\title{
Progression of hydrocephalus during corticosteroid therapy for neurosarcoidosis
}

\author{
Kevin T. Foley, Joel D. Howell and Larry Junck \\ Department of Internal Medicine and the Department of Neurology, University of Michigan, Michigan, USA.
}

\begin{abstract}
Summary: We describe a patient with sarcoid meningitis and hydrocephalus who improved rapidly after initiation of oral prednisone therapy, but who later decompensated acutely and required an emergency ventriculoperitoneal shunt. Hydrocephalus associated with neurosarcoidosis may progress despite steroid treatment, even when symptoms have improved. If hydrocephalus associated with neurosarcoidosis is treated with corticosteroids and without a shunt procedure we suggest that the corticosteroids should be continued at high doses for a prolonged period.
\end{abstract}

\section{Introduction}

Sarcoidosis is a chronic inflammatory disease of unknown aetiology. Non-caseating granulomas may involve any organ system, including the central and peripheral nervous systems. ${ }^{1-5}$ Hydrocephalus is found in approximately $10 \%$ of patients with neurosarcoidosis ${ }^{2-4}$ and is usually of poor prognostic significance. ${ }^{2,3,6-8}$ The management of hydrocephalus associated with neurosarcoidosis has centred on treatment of the underlying disease with corticosteroids; however, the response to steroid therapy is unpredictable and often incomplete. ${ }^{1-3,9,10}$ Cerebrospinal fluid shunting is usually indicated if hydrocephalus is associated with severe or life-threatening symptoms, but apart from these circumstances the role of shunting has not been defined.

\section{Case report}

A 33 year old, right-handed black woman presented to the University of Michigan in January 1987 complaining of recurrent bitemporal headaches and bloody nasal discharge for several months.

Sarcoidosis had been originally diagnosed elsewhere in 1982 on the basis of liver and salivary gland biopsies during an evaluation for abdominal pain. Treatment with prednisone at a dose of 10 to $30 \mathrm{mg} /$ day had been associated with symptomatic

Correspondence: J. D. Howell, M.D., Ph.D., Division of General Medicine, Taubman Center 3116-0376, University of Michigan Medical Center, 1500 E. Medical Center Drive, Ann Arbor, MI 48109-0376, USA.

Accepted: 17 February 1989 improvement. The patient was admitted to another hospital in December 1986, for evaluation of headache, dizziness, and one episode of loss of consciousness over the preceeding 4 months. Computed tomography (CT) of the head showed minimally enlarged lateral ventricles and a prominent fourth ventricle. Prednisone therapy was continued at $10 \mathrm{mg} /$ day.

On presentation she was markedly bradykinetic, but not rigid, and her nasal mucosae were covered with mucopurulent material. Sinus radiographs showed fluid in the right maxillary sinus. A diagnosis of sinusitis was made, and trimethoprim/sulfamethoxazole was prescribed.

Four days later, the patient became disoriented to place and exhibited perseverating behaviour followed by a tonic-clonic seizure and 3 days later she returned complaining of headache, blurred vision, and neck stiffness. Neurological examination was unchanged except for nuchal rigidity and difficulty with immediate and recent memory and calculations. Lumbar puncture revealed an opening pressure of $30 \mathrm{~cm}$ cerebrospinal fluid (CSF). The CSF appeared clear and contained 7 red blood cells and 34 white blood cells per cubic millimeter, of which 29 were lymphocytes and 5 were polymorphonuclear cells. The CSF protein was $1.2 \mathrm{~g} / \mathrm{l}$, and the glucose was $2.5 \mathrm{mmol} / 1$ with a concomitant serum glucose of $5.2 \mathrm{mmol} / \mathrm{l}$. CSF cytology and cryptococcal antigen were negative, as were cultures for bacteria, fungi and acid-fast bacilli. CT scan showed marked communicating hydrocephalus with leptomeningeal enhancement of the basal cisterns and cerebral gyri and sulci (Figure 1). Prednisone was increased to 


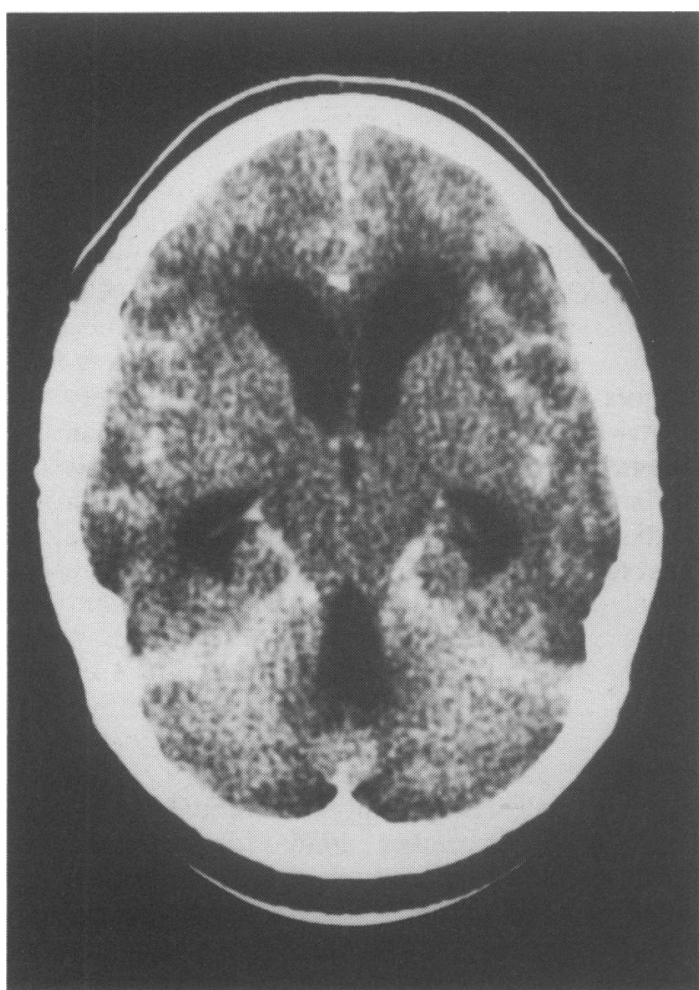

Figure 1 Computed tomographic scan with contrast demonstrating communicating hydrocephalus and abnormal enhancement of the cerebral convexities.

$60 \mathrm{mg}$ daily with improvement of the patient's headache over the next 7 days. Although a repeat CT scan one month after presentation showed no change in the hydrocephalus, prednisone was decreased to $40 \mathrm{mg}$ daily because of her striking clinical improvement.

The patient reported only occasional mild headaches and her bradykinesia had resolved. Prednisone was reduced to $20 \mathrm{mg}$ daily one month after the repeat CT scan. Audiogram, brain stem auditory evoked potentials, and ophthalmological examination including Humphrey visual fields were normal.

After experiencing 2 days of severe headaches, she presented in extremis at the time of a scheduled appointment 10 days later. When supine, she developed laboured respiration, confusion, dysconjugate gaze, and decerebrate posturing, all of which returned to normal with return to the sitting position. The optic discs were pale bilaterally. She exhibited generalized hyperreflexia with flexor plantar responses. CT revealed increased dilatation of the lateral and fourth ventricles (Figure 2).

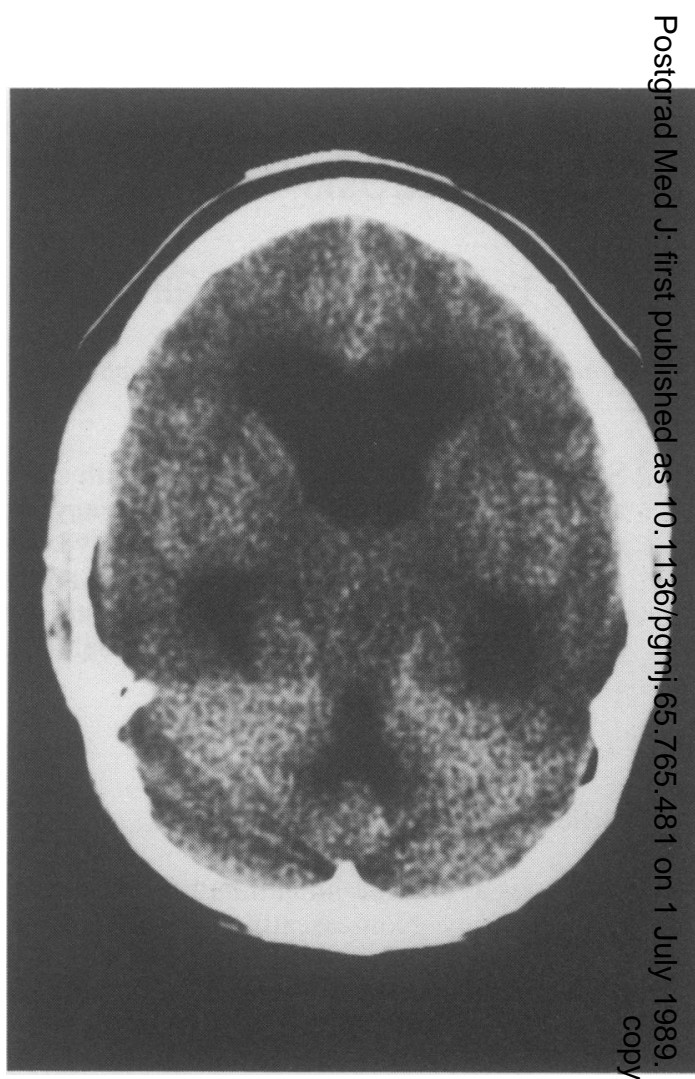

ํㅡ. 뭉

Figure 2 Non-contrast computed tomographię san showing increased hydrocephalus at the time of the emergency shunt procedure.

A right ventriculoperitoneal shunt was performed as an emergency, and she improved rapidly. A CT sean 2 weeks later demonstrated complete decompresșison of the right ventricle with minimal reduction on theteft and an unchanged fourth ventricle. After surgery, 色er prednisone dosage was gradually tapered to $20 \mathrm{mg}$ every other day. She continued to do well, witliut change on follow-up head CT studies, until January 1988, when she again developed moderate to severe headaches and blurred vision. Visual fields and açity were normal, and the fundi again revealed atrophy of the optic discs. Neurological examination revesed right sixth nerve palsy and the return of generalized bradykinesia. Non-contrast head CT on January 27 showed normal size ventricles. Lumbar punctire revealed an opening pressure of $22 \mathrm{~cm} \mathrm{CSF}$ and clesar, xanthochromic fluid with zero red blood cells an $\$ 44$ white blood cells per $\mathrm{mm}^{3}$ of which $94 \%$ were $18 \mathrm{~m}$ phocytes. The CSF protein was $5.9 \mathrm{~g} / 1$ and the glucose $2.0 \mathrm{mmol} / \mathrm{l}$. CSF angiotensin converting enztme (ACE) was $0.1 \mathrm{U} / \mathrm{ml}$. Prednisone was increasedoto $40 \mathrm{mg}$ every other day with improvement of $\overline{\mathrm{T}}$ er 
symptoms over the following week. Since that time, she has required revision of her shunt on two occasions; once in May 1988, due to the formation of an abdominal pseudocyst, and again in June 1988, after developing a shunt infection. Apart from mild residual memory deficits, her clinical and radiographic response has been maintained.

\section{Discussion}

The treatment of neurosarcoidosis with prednisone was first described in $1957,{ }^{11}$ and corticosteroids have since remained the primary therapeutic agents. Surgery is generally performed only for acute or unremitting neurological compromise due to obstruction in the CSF pathways. ${ }^{12-14}$ There are no controlled trials describing the efficacy of any mode of treatment, but the bulk of evidence suggests that prolonged steroid therapy can control many of the manifestations of neurosarcoidosis. ${ }^{1-4,8,9,15}$ Decisions regarding steroid therapy are difficult because treatment failures occur frequently ${ }^{1,2,5,15,16}$ and long courses of steroids incur significant undesirable side effects. Furthermore, although neurosarcoidosis usually runs a chronic course, spontaneous improvement and remission occur in a number of cases, ${ }^{1-3,5}$ and sustained improvement has been documented with both short courses of therapy ${ }^{1,7}$ and pulsed intravenous steroid treatment. ${ }^{17}$ Except for CT and magnetic resonance imaging (MRI) studies, ${ }^{18-22}$ reliable indices by which to follow disease activity are lacking, which can make management decisions even more difficult.

Treatment strategies for hydrocephalus cannot be easily formulated from a review of the literature due to dissimilarities in treatment methods, types of neurosarcoid involvement, and the lack of details in many reports. Plausible treatment options include: (1) early shunting, at the diagnosis of hydrocephalus; (2) steroid therapy, with shunting if the ventricles fail to decrease in size on CT or MRI; and (3) steroid therapy, with shunting if the symptoms fail to improve. However, hydrocephalus associated with neurosarcoidosis has not, to our knowledge, been reported to respond in a sustained manner to medical therapy with corticosteroids exclusively. Furthermore, the progression

\section{References}

1. Silverstein, A., Feuer, M.M. \& Siltzbach, L.E. Neurologic sarcoidosis. Arch Neurol 1965, 12: 1-11.

2. Oksanen, V. Neurosarcoidosis: clinical presentations and course in 50 patients. Acta Neurol Scand 1986, 73: 283-290.

3. Delaney, P. Neurologic manifestations in sarcoidosis: review of the literature with a report of 23 cases. Ann Intern Med 1977, 87: 336-345. of our patient's hydrocephalus despite clinical improvement suggests that the last approach involves considerable risk. It is possible that the improvement in her symptoms of headache and neck stiffness was due to improvement in her concomitant sarcoid meningitis without control of her hydrocephalus. This case illustrates the difficulties in relying on clinical features to guide treatment of neurosarcoidosis.

We propose the following guidelines for management of hydrocephalus in neurosarcoidosis. The physician should determine whether the hydrocephalus is compensated or uncompensated. Patients with compensated hydrocephalus lack headaches, lethargy, papilloedema and a progressive disorder of gait; if lumbar puncture is performed, opening pressure is normal. Patients with uncompensated hydrocephalus that is acute or accompanied by severe symptoms should undergo early shunt placement, in addition to steroid therapy at moderate to high doses. For patients with uncompensated subacute or chronic hydrocephalus accompanied by mild or moderate symptoms, treatment may be initiated either with high dose steroid therapy alone or with shunt placement and moderate steroid doses. Patients with hydrocephalus being treated with steroid therapy alone should be followed closely with serial neurological examinations and CT or MRI scans. High dose treatment should be continued until decreased ventricular size is documented by follow-up imaging studies, or, if clinical improvement occurs in the absence of a decrease in ventricular size, for a minimum of several months. If the symptoms prove refractory, or if progressive ventricular enlargement is documented, insertion of a shunt is recommended. For patients thought to have compensated hydrocephalus, treatment should be based on their current symptoms, but periodic examinations and imaging studies should be performed.

\section{Acknowledgements}

We would like to express our appreciation to Barb Inman and Sonja Brandon-Dabney for assistance in preparing this manuscript.

4. Stern, B.J., Krumholz, A., Johns, C., Scott, P. \& Nissim, J. Sarcoidosis and its neurologic manifestations. Arch Neurol 1985, 42: 909-917.

5. Wiederholt, W.C. \& Siekert, R.G. Neurologic manifestations of sarcoidosis. Neurology (Minneap) 1965, 15: 1147-1154. 
6. Schlitt, M.S., Duvall, E.R., Bonnin, J. \& Morawetz, R.B. Neurosarcoidosis causing ventricular loculation, hydrocephalus, and death. Surg Neurol 1986, 26: 67-71.

7. Lukin, R.R., Chambers, A.A. \& Soleimanpour, M. Outlet obstruction of the fourth ventricle in sarcoidosis. Neuroradiology 1975, 10: 65-68.

8. Luke, R.A., Stern, B.J., Krumholz, A. \& Johns, C.J. Neurosarcoidosis: the long-term clinical course. Neurology 1987, 37: 461-463.

9. Matthews, W.B. Sarcoidosis of the nervous system. $J$ Neurol Neurosurg Psychiat 1965, 28: 23-29.

10. Waxman, J.S. \& Sher, J.H. The spectrum of central nervous system sarcoidosis: a clinical and pathologic study. Mt Sinai J Med 1979, 46: 309-317.

11. Fitzpatrick, D.P. \& Ewart, G.E. Central nervous system sarcoidosis successfully treated with prednisone. Arch Int Med 1957, 100: 139-142.

12. Cahill, D.W. \& Salcman, M. Neurosarcoidosis: a review of the rarer manifestations. Surg Neurol 1981, 15: 204-211.

13. Ho, S.U., Berenberg, R.A., Kim, K.S. \& DalCanto, M.C. Sarcoid encephalopathy with diffuse inflammation and focal hydrocephalus shown by sequential CT. Neurology 1979, 29: 1161-1165.

14. Whelan, M.A. \& Stern, J.S. Sarcoidosis presenting as a posterior fossa mass. Surg Neurol 1981, 15: 455-457.
15. Douglas, A.C. \& Maloney, A.F.J. Sarcoidosis of the central nervous system. J Neurol Neurosurg Psychiåึ 1973, 36: 1024-1033.

16. Manz, H.J. Pathobiology of neurosarcoidosis and clinicopathologic correlation. Can J Neurol Sci 1983, 10 ? $50-55$.

17. Lundh, T. \& Wikkelso, C. Sarcoidosis with hyd $\overrightarrow{\overline{\vec{\digamma}}}$ rocephalus: report of a case successfully treated with ventriculo-peritoneal shunt and methylprednisolone pulse therapy. Acta Neurol Scand 1987, 76: 365-368.

18. Bahr, A.L., Krumholz, A., Kristt, D. \& Hodges, F.J Neuroradiological manifestations of intracranial sar尺 coidosis. Radiology 1978, 127: 713-717.

19. Kendall, B.E. \& Tatler, G.L.V. Radiological findings in neurosarcoidosis. Br J Radiol 1978, 51: 81-92.

20. Greco, A. \& Steiner, R.E. Magnetic resonance imaging in neurosarcoidosis. Magn Reson Imaging 1987, 15-21.

21. Ketonen, L., Oksanen, V. \& Kuuliala, I. Prelimina experience of magnetic resonance imaging in neurosar:coidosis. Neuroradiology 1987, 29: 127-129.

22. Reed, L.D., Abbas, S., Markivee, C.R. \& Fletcher, J.W Neurosarcoidosis responding to steroids. AJR 1986, 146 819-821. 